The Quarterly Journal of Mathematics

Quart. J. Math. 00 (2017), 1-14; doi:10.1093/qmath/hax003

\title{
THE COCYCLE IDENTITY HOLDS UNDER STOPPING
}

\author{
by ALEXANDER C. R. BELTON ${ }^{\dagger}$ \\ (Department of Mathematics and Statistics, Lancaster University, UK) \\ and KALYAN B. SINHA \\ (Jawaharlal Nehru Centre for Advanced Scientific Research, Bangalore, India)
}

[Received 14 December 2016]

\begin{abstract}
In recent work of the authors, it was shown how to use any finite quantum stop time to stop the CCR flow and its strongly continuous isometric cocycles (Q. J. Math. 65:1145-1164, 2014). The stopped cocycle was shown to satisfy a stopped form of the cocycle identity, valid for deterministic increments of the time used for stopping. Here, a generalization of this identity is obtained, where both cocycle parameters are replaced with finite quantum stop times.
\end{abstract}

\section{Introduction}

The history of stopping times in non-commutative probability begins in 1979, with Hudson's work on stopping canonical Wiener processes [4]. Since then, many authors have contributed to the subject, and it has developed in various directions and settings: abstract von Neumann algebras, to produce first exit times in $C^{*}$ algebras and to stop quantum stochastic integrals, for example. A good introduction for the latter is provided by [5]; see [2] for further references.

In this note, we extend a previous result [2, Theorem 7.2], which itself built upon work of Parthasarathy and Sinha [7] and Applebaum [1]. Let $V$ be a strongly continuous isometric cocycle of the CCR flow $\sigma$, so that

$$
V_{s+t}=\widehat{V}_{s} \sigma_{s}\left(V_{t}\right) \quad \text { for all } s, t \in \mathbb{R}_{+},
$$

where $\widehat{V}$ is the identity-adapted projection of the $p$-adapted process $V$. The importance of this identity in classical and quantum probability is well known; it has an intimate connection with stochastic integral representation and Feynman-Kac formulae [3, 8].

If $S$ is a finite quantum stop time, then Theorem 7.2 of [2] gives the stopped cocycle identity

$$
V_{S+t}=\widehat{V}_{S} \sigma_{S}\left(V_{t}\right) \quad \text { for all } t \in \mathbb{R}_{+} \text {. }
$$

It is shown below that the following generalization of (1) holds: if $T$ is another finite quantum stop time and the CCR flow $\sigma$ has countable rank, then

\footnotetext{
†Corresponding author. E-mail: a.belton@lancaster.ac.uk

${ }^{\ddagger}$ E-mail: kbs@jncasr.ac.in
} 


$$
V_{S \star T}=\widehat{V}_{S} \sigma_{S}\left(V_{T}\right),
$$

where $S \star T$ is the convolution of $S$ and $T$. If $V$ acts on an initial space h, it follows that setting

$$
\alpha_{S}(a)=V_{S}(a \otimes I) V_{S}^{*} \quad \text { for all } a \in B(\mathrm{~h})
$$

gives a generalized Evans-Hudson flow $\alpha_{S}$ which satisfies a non-deterministic version of the mapping-cocycle relation,

$$
\alpha_{S \star T}=\widehat{\alpha}_{S} \circ \sigma_{S} \circ \alpha_{T}
$$

A related result was obtained by Hudson [6]; his work considers two-parameter Weyl processes, which are composed of unitary operators acting on a trivial initial space, and requires the stopping times to obey a strong form of ordering.

Below, the notation of [2] is followed throughout. In particular, the algebraic tensor product is denoted by $\underline{\otimes}$, with $\otimes$ the Hilbert-space and $\bar{\otimes}$ the ultraweak product.

\section{Stopped maps with a non-trivial initial space}

In Sections 6 and 7 of [2], certain maps $E_{S}, \Gamma_{S}$ and $\sigma_{S}$ are extended to the case of a non-trivial initial space, so that the ambient Fock space $\mathcal{F}$ is replaced by $\mathrm{h} \otimes \mathcal{F}$, where $\mathrm{h}$ is a complex Hilbert space. In order to familiarize the reader with key ideas and notation from [2], and as the construction of these extensions is not quite immediate, the details are provided in this section, together with some further observations.

Notation 2.1 Let $\mathcal{F}=\Gamma_{+}\left(L^{2}\left(\mathbb{R}_{+} ; \mathrm{k}\right)\right)$ be Boson Fock space over the complex Hilbert space of square-integrable functions on the half line $\mathbb{R}_{+}:=[0, \infty)$, with values in the complex Hilbert space k. Recall the tensor-product decomposition $\mathcal{F}=\mathcal{F}_{t)} \otimes \mathcal{F}_{[t}$, valid for all $t \in(0, \infty)$, where

$$
\mathcal{F}_{t)}:=\Gamma_{+}\left(L^{2}([0, t) ; \mathrm{k})\right) \quad \text { and } \quad \mathcal{F}_{[t}:=\Gamma_{+}\left(L^{2}([t, \infty) ; \mathrm{k})\right) \text {, }
$$

given by extending the identification of exponential vectors such that $\varepsilon(f)=\varepsilon\left(\left.f\right|_{[0, t)}\right) \otimes \varepsilon\left(\left.f\right|_{[t, \infty)}\right)$ for all $f \in L^{2}\left(\mathbb{R}_{+} ; \mathrm{k}\right)$. Let $I, I_{t)}$ and $I_{[t}$ denote the identity operators on $\mathcal{F}, \mathcal{F}_{t)}$ and $\mathcal{F}_{[t}$, respectively, and let $\mathcal{E}$ denote the linear span of the set of exponential vectors in $\mathcal{F}$.

Definition 2.2 Let $S$ be a finite quantum stop time, so that $S: \mathcal{B}[0, \infty] \rightarrow B(\mathcal{F})$ is a map from the Borel subsets of the extended half line to the set of orthogonal projections on $\mathcal{F}$, such that

(1) the map $A \mapsto\langle x, S(A) y\rangle$ is a complex measure for all $x, y \in \mathcal{F}$,

(2) the total mass $S([0, \infty])=I$, with $S(\{\infty\})=0$, and

(3) identity adaptedness holds, so that $S(\{0\})=0$ and $S([0, t]) \in B\left(\mathcal{F}_{t)}\right) \otimes I_{[t}$ for all $t \in(0, \infty)$.

Notation 2.3 For all $t \in \mathbb{R}_{+}$, let $E_{t}:=\Gamma_{+}\left(1_{[0, t)}\right) \in B(\mathcal{F})$ be the second quantization of the operator obtained by letting this indicator function act by multiplication, so that $E_{t}$ is the orthogonal projection onto $\mathcal{F}_{t)} \otimes \varepsilon\left(\left.0\right|_{[t, \infty)}\right)$, and let $E_{\infty}:=I$. 
Proposition 2.4 Let $\pi=\left\{0=\pi_{0}<\cdots<\pi_{n+1}=\infty\right\}$ be a finite partition of $[0, \infty]$ and let $\mathrm{h}$ be a complex Hilbert space. If $\widetilde{E}_{S, \pi}:=I_{\mathrm{h}} \otimes E_{S, \pi}$, where

$$
E_{S, \pi}:=\sum_{j=1}^{n+1} S\left(\left(\pi_{j-1}, \pi_{j}\right]\right) E_{\pi_{j}}
$$

then $E_{S, \pi} \rightarrow E_{S}$ and $\widetilde{E}_{S, \pi} \rightarrow \widetilde{E}_{S}:=I_{\mathrm{h}} \otimes E_{S}$ in the strong operator topology as $\pi$ is refined, where $E_{S}$ and $\widetilde{E}_{S}$ are orthogonal projections.

Proof. The proof of [2, Theorem 3.7] gives that $E_{S, \pi} \rightarrow E_{S}$ strongly on $\mathcal{E}$, and thus $\widetilde{E}_{S, \pi} \rightarrow \widetilde{E}_{S}$ strongly on $\mathrm{h} \underline{\otimes} \mathcal{E}$; the result follows by the density of this last space in $\mathrm{h} \otimes \mathcal{F}$.

Notation 2.5 For all $s \in \mathbb{R}_{+}$, let $\Gamma_{s}:=\Gamma_{+}\left(\theta_{s}\right) \in B(\mathcal{F})$ be the second quantization of the isometric right shift, such that $\left(\theta_{s} f\right)(t)=1_{[s, \infty)}(t) f(t-s)$ for all $t \in \mathbb{R}_{+}$, and let $\Gamma_{\infty}:=E_{0}$.

Proposition 2.6 Let $\pi$ and $\mathrm{h}$ be as in Proposition 2.4. If $\widetilde{\Gamma}_{S, \pi}:=I_{\mathrm{h}} \otimes \Gamma_{S, \pi}$, where

$$
\Gamma_{S, \pi}:=\sum_{j=1}^{n+1} S\left(\left(\pi_{j-1}, \pi_{j}\right]\right) \Gamma_{\pi_{j}},
$$

then $\Gamma_{S, \pi} \rightarrow \Gamma_{S}$ and $\widetilde{\Gamma}_{S, \pi} \rightarrow \widetilde{\Gamma}_{S}:=I_{\mathrm{h}} \otimes \Gamma_{S}$ in the strong operator topology as $\pi$ is refined, where $\Gamma_{S}$ and $\widetilde{\Gamma}_{S}$ are isometries.

Proof. The claims about $\Gamma_{S, \pi}$ and $\Gamma$ follow from the proof of [2, Theorem 3.8], which also gives that

$$
\left\|\left(\widetilde{\Gamma}_{S, \pi}-\widetilde{\Gamma}_{S}\right) u \otimes x\right\|=\|u\|\left\|\left(\Gamma_{S, \pi}-\Gamma_{S}\right) x\right\| \rightarrow 0
$$

as $\pi$ is refined, for all $u \in \mathrm{h}$ and $x \in \mathcal{F}$. As $\Gamma_{S, \pi}$ and $\Gamma_{S}$ are isometries, the same is true for $\widetilde{\Gamma}_{S, \pi}$ and $\widetilde{\Gamma}_{S}$. Thus, $\widetilde{\Gamma}_{S, \pi} \rightarrow \widetilde{\Gamma}_{S}$ strongly on $\mathrm{h} \underline{\otimes} \mathcal{F}$, and so on $\mathrm{h} \otimes \mathcal{F}$, since $\left\|\widetilde{\Gamma}_{S, \pi}\right\|=1$ for all $\pi$.

Notation 2.7 For all $t \in \mathbb{R}_{+}$, let the ultraweakly continuous unital $*$-homomorphism

$$
\sigma_{t}: B(\mathcal{F}) \rightarrow B(\mathcal{F}) ; \quad X \mapsto I_{t)} \otimes \Gamma_{t} X \Gamma_{t}^{*},
$$

where $\Gamma_{t}$ is regarded here as an isometric isomorphism from $\mathcal{F}$ to $\mathcal{F}_{t t}$ with inverse $\Gamma_{t}^{*}$, and let $\widetilde{\sigma}_{t}:=\mathrm{id}_{B(\mathrm{~h})} \bar{\otimes} \sigma_{t}$. Recall that $\left(\sigma_{t}: t \in \mathbb{R}_{+}\right)$is the CCR flow semigroup with rank dim $\mathrm{k}$.

Notation 2.8 Let $\mathcal{F}_{S)}:=E_{S}(\mathcal{F})$ and $\mathcal{F}_{[S}:=\Gamma_{S}(\mathcal{F})$ be the pre- $S$ and post- $S$ spaces, with identity operators $I_{S)}$ and $I_{[S}$, respectively.

Theorem 2.9 Let $\mathrm{h}$ and $\pi$ be as in Proposition 2.4. If

$$
\widetilde{\sigma}(Z)_{S, \pi}:=\left(\mathrm{id}_{B(\mathrm{~h})} \bar{\otimes} \sigma_{S, \pi}\right)(Z) \quad \text { for all } Z \in B(\mathrm{~h} \otimes \mathcal{F}),
$$

where 


$$
\sigma_{S, \pi}: B(\mathcal{F}) \rightarrow B(\mathcal{F}) ; X \mapsto \sum_{j=1}^{n+1} \sigma_{\pi_{j}}(X) S\left(\left(\pi_{j-1}, \pi_{j}\right]\right)
$$

then $\sigma_{S, \pi} \rightarrow \sigma_{S}$ and $\widetilde{\sigma}_{S, \pi} \rightarrow \widetilde{\sigma}_{S}:=\mathrm{id}_{B(\mathrm{~h})} \bar{\otimes} \sigma_{S}$ pointwise in the strong operator topology as $\pi$ is refined, where $\sigma_{S}$ and $\widetilde{\sigma}_{S}$ are ultraweakly continuous unital $*$-homomorphisms. Furthermore, there exist isometric isomorphisms

$$
j_{S}: \mathcal{F}_{S)} \otimes \mathcal{F}_{[S} \rightarrow \mathcal{F} \quad \text { and } \quad \widetilde{J}_{S}: \mathcal{F}_{S)} \otimes \mathrm{h} \otimes \mathcal{F}_{[S} \rightarrow \mathrm{h} \otimes \mathcal{F}
$$

such that

$$
\begin{array}{cc} 
& \left.\sigma_{S}(X)=j_{S}\left(I_{S}\right) \otimes \Gamma_{S} X \Gamma_{S}^{*}\right) j_{S}^{*} \quad \text { for all } X \in B(\mathcal{F}) \\
\text { and } \quad \widetilde{\sigma}_{S}(Z)=\widetilde{J}_{S}\left(I_{S} \otimes \widetilde{\Gamma}_{S} Z \widetilde{\Gamma}_{S}^{*}\right) \widetilde{J}_{S}^{*} \quad \text { for all } Z \in B(\mathrm{~h} \otimes \mathcal{F}) .
\end{array}
$$

Proof. The convergence of $\sigma_{S, \pi}$ to $\sigma_{S}$, and the fact that the latter is a unital *-homomorphism, follows from [2, Theorem 5.2]. The representation (2) is [2, Proposition 5.3], and this shows that the map $X \mapsto \sigma_{S}(X)$ is continuous when $B(\mathcal{F})$ is equipped with the ultraweak topology, as ampliation gives a normal representation of any von Neumann algebra. In particular, the map $\widetilde{\sigma}_{S}$ is an ultraweakly continuous unital $*$-homomorphism such that (3) holds, where the isometric isomorphism

$$
\widetilde{J}_{S}: \mathcal{F}_{S)} \otimes \mathrm{h} \otimes \mathcal{F}_{[S} \rightarrow \mathrm{h} \otimes \mathcal{F} ; x \otimes u \otimes y \mapsto u \otimes j_{S}(x \otimes y),
$$

because (3) holds if $Z$ is a simple tensor, and both sides are ultraweakly continuous functions of $Z$.

It remains to prove that $\widetilde{\sigma}_{S, \pi}$ converges to $\widetilde{\sigma}_{S}$. Working as in the proof of [2, Theorem 5.2], if the finite partition $\pi^{\prime}$ is a refinement of $\pi$, then, for any $u \in \mathrm{h}$ and any $f \in L^{2}\left(\mathbb{R}_{+}\right.$; k) with compact support,

$$
\begin{aligned}
& \left\|\left(\widetilde{\sigma}_{S, \pi^{\prime}}-\widetilde{\sigma}_{S, \pi}\right)(Z) u \varepsilon(f)\right\| \\
& \quad \leq\left\|S\left(\left[0, \pi_{n}\right]\right) \varepsilon(f)\right\| \sup \left\{\|\left(\widetilde{\sigma}_{r}(Z)-Z\right) u \varepsilon(f(\cdot+s)): r \in\left[0, \delta_{\pi}\right], s \in[0, \tau]\right\} \\
& \quad+\left\|S\left(\left(\pi_{n}, \infty\right)\right) u \varepsilon(f)\right\|(\|Z\|+1),
\end{aligned}
$$

where $\delta_{\pi}:=\max \left\{\pi_{j}-\pi_{j-1}: j=1, \ldots, n\right\}$ and $f$ has support contained in $[0, \tau] \subseteq[0, \infty)$.

Using the same argument as in the proof of [2, Theorem 5.2], and noting that $r \mapsto \widetilde{\sigma}_{r}(Z)$ is strongly continuous, it now follows that $\widetilde{\sigma}_{S, \pi}(Z) u \varepsilon(f)$ is convergent, as $\pi$ is refined, for any $u \in \mathrm{h}$ and any $f \in L^{2}\left(\mathbb{R}_{+} ; \mathrm{k}\right)$ with compact support; let the limit be denoted by $\lambda_{S}(Z) u \varepsilon(f)$ and extend by linearity. Since

$$
\left\|\lambda_{S}(Z) z\right\|=\lim _{\pi}\left\|\widetilde{\sigma}_{S, \pi}(Z) z\right\| \leq\|Z\|\|z\| \quad \text { for all } z \in \mathrm{h} \underline{\otimes} \mathcal{E}_{c},
$$

where $\mathcal{E}_{c}$ is the linear span of those exponential vectors corresponding to functions with compact support, there exists a bounded linear operator $\lambda_{S}(Z)$ on $\mathrm{h} \otimes \mathcal{F}$ which extends the linear map 
$z \mapsto \lambda_{S}(Z) z$. Furthermore, the usual approximation argument gives that $\widetilde{\sigma}_{S, \pi}(Z) \rightarrow \lambda_{S}(Z)$ in the strong operator topology, everywhere on $\mathrm{h} \otimes \mathcal{F}$.

To conclude, we use (3) and argue as the proof of [2, Proposition 5.3]. Using the notation of that proof and the identity at the top of [2, p. 1158], that

$$
\left\langle I_{S, \pi \cap[0, t]}(f, \Gamma \varepsilon(g)), \sigma_{S, \pi}(X) I_{S, \pi \cap[0, t]}\left(f^{\prime}, \Gamma \varepsilon\left(g^{\prime}\right)\right)\right\rangle=\left\langle E_{S, \pi \cap[0, t]} \varepsilon(f), E_{S, \pi \cap[0, t]} \varepsilon\left(f^{\prime}\right)\right\rangle\left\langle\varepsilon(g), X \varepsilon\left(g^{\prime}\right)\right\rangle,
$$

together with the ultraweak continuity of $\widetilde{\sigma}_{S, \pi}$, it follows that

$$
\begin{aligned}
\langle u & \left.\otimes I_{S, \pi \cap[0, t]}(f, \Gamma \varepsilon(g)), \widetilde{\sigma}_{S, \pi}(Z) u^{\prime} \otimes I_{S, \pi \cap[0, t]}\left(f^{\prime}, \Gamma \varepsilon\left(g^{\prime}\right)\right)\right\rangle \\
& =\left\langle E_{S, \pi \cap[0, t]} \varepsilon(f), E_{S, \pi \cap[0, t]} \varepsilon\left(f^{\prime}\right)\right\rangle\left\langle u \varepsilon(g), Z u^{\prime} \varepsilon\left(g^{\prime}\right)\right\rangle
\end{aligned}
$$

for all $u, u^{\prime} \in \mathrm{h}, f, f^{\prime}, g, g^{\prime} \in L^{2}\left(\mathbb{R}_{+} ; \mathrm{k}\right), t \in(0, \infty)$ and $Z \in B(\mathrm{~h} \otimes \mathcal{F})$. As $\pi$ is refined, the right-hand side converges to

$$
\left.\left\langle E_{S, t} \varepsilon(f) \otimes u \otimes \Gamma_{S} \varepsilon(g),\left(I_{S}\right) \otimes \widetilde{\Gamma}_{S} Z \widetilde{\Gamma}_{S}^{*}\right) E_{S, t} \varepsilon\left(f^{\prime}\right) \otimes u^{\prime} \otimes \Gamma_{S} \varepsilon\left(g^{\prime}\right)\right\rangle,
$$

by [2, Theorem 3.7], whereas the left-hand side converges to

$$
\begin{aligned}
\langle u & \left.\otimes j_{S}\left(E_{S, t} \varepsilon(f) \otimes \Gamma_{S} \varepsilon(g)\right), \lambda_{S}(Z) u^{\prime} \otimes j_{S}\left(E_{S, t} \varepsilon\left(f^{\prime}\right) \otimes \Gamma_{S} \varepsilon\left(g^{\prime}\right)\right)\right\rangle \\
& =\left\langle\widetilde{J}_{S}\left(E_{S, t} \varepsilon(f) \otimes u \otimes \Gamma_{S} \varepsilon(g)\right), \lambda_{S}(Z) \widetilde{J}_{S}\left(E_{S, t} \varepsilon\left(f^{\prime}\right) \otimes u^{\prime} \otimes \Gamma_{S} \varepsilon\left(g^{\prime}\right)\right)\right\rangle,
\end{aligned}
$$

by [2, Lemma 3.4 and Theorem 3.10]. The result follows.

REMARK 2.10 The representations (2) and (3) also give that $X \mapsto \sigma_{S}(X)$ and $Z \mapsto \widetilde{\sigma}_{S}(Z)$ are continuous on bounded subsets of $B(\mathcal{F})$ and $B(\mathrm{~h} \otimes \mathcal{F})$, respectively, when these spaces are equipped with the strong operator topology, since this is true of the ampliation map $T \mapsto I \otimes T$.

\section{The cocycle identity with two stop times}

Definition 3.1 ([2, Definition 4.1]) The convolution $S \star T$ of two finite quantum stop times $S$ and $T$ is

$$
S \star T: \mathcal{B}\left(\mathbb{R}_{+}\right) \rightarrow B(\mathcal{F}) ; A \mapsto(S \otimes T)\left(f^{-1}(A)\right),
$$

where

$$
f: \mathbb{R}_{+} \times \mathbb{R}_{+} \rightarrow \mathbb{R}_{+} ;(x, y) \mapsto x+y
$$

and

$$
S \otimes T: \mathcal{B}\left(\mathbb{R}_{+} \times \mathbb{R}_{+}\right) \rightarrow B(\mathcal{F}) ; A \times B \mapsto j_{S}\left(\left.S(A)\right|_{\left.\mathcal{F}_{S}\right)} \otimes \Gamma_{S} T(B) \Gamma_{S}^{*}\right) j_{S}^{*}
$$


Lemma 3.2 Let $S$ and $T$ be finite quantum stop times. Then

$$
(S \otimes T)(A \times B)=S(A) \sigma_{S}(T(B)) \quad \text { for all } A, B \in \mathcal{B}[0, \infty] .
$$

Proof. If $t \in[0, \infty]$ then, by Theorems 3.7 and 3.10, together with Lemma 3.4 of [2],

$$
\begin{aligned}
j_{S}\left(S([0, t]) E_{S} \varepsilon(f) \otimes \Gamma_{S} x\right)=j_{S}\left(E_{S, t} \varepsilon(f) \otimes \Gamma_{S} x\right) & =\int_{[0, t]} S(\mathrm{~d} s) \varepsilon\left(\left.f\right|_{[0, s)}\right) \otimes \Gamma_{s} x \\
& =S([0, t]) \int_{[0, \infty]} S(\mathrm{~d} s) \varepsilon\left(\left.f\right|_{[0, s)}\right) \otimes \Gamma_{S} x \\
& =S([0, t]) j_{S}\left(E_{S} \varepsilon(f) \otimes \Gamma_{S} x\right)
\end{aligned}
$$

for all $f \in L^{2}\left(\mathbb{R}_{+} ; \mathrm{k}\right)$ and $x \in \mathcal{F}$. Hence

$$
j_{S}\left(\left.S(A)\right|_{\left.\mathcal{F}_{S}\right)} \otimes I_{[S}\right) j_{S}^{*}=S(A) \quad \text { for all } A \in \mathcal{B}[0, \infty] .
$$

It follows that

$$
\begin{aligned}
(S \otimes T)(A \times B) & :=j_{S}\left(\left.S(A)\right|_{\left.\mathcal{F}_{S}\right)} \otimes \Gamma_{S} T(B) \Gamma_{S}^{*}\right) j_{S}^{*} \\
& =j_{S}\left(\left.S(A)\right|_{\left.\mathcal{F}_{S}\right)} \otimes I_{[S}\right) j_{S}^{*} j_{S}\left(I_{S} \otimes \Gamma_{S} T(B) \Gamma_{S}^{*}\right) j_{S}^{*}=S(A) \sigma_{S}(T(B))
\end{aligned}
$$

for all $A, B \in \mathcal{B}[0, \infty]$, where the final identity is a consequence of (2).

\section{REMARK 3.3}

(1) If the quantum stop times $S$ and $T$ are extended by ampliation to act on $\mathrm{h} \otimes \mathcal{F}$, then the identity (4) becomes

$$
(S \otimes T)(A \times B)=S(A) \widetilde{\sigma}_{S}(T(B)) \quad \text { for all } A, B \in \mathcal{B}[0, \infty]
$$

This extension will be made when appropriate without further comment.

(2) If $0 \leq p<q<\infty$ and $0 \leq r<s<\infty$, then Theorem 2.9 implies that

$$
\begin{aligned}
(S \otimes T)((p, q] \times(r, s]) & =S((p, q]) \widetilde{\sigma}_{S}(T((r, s])) \\
& =\operatorname{st}_{\pi} \lim _{\pi} \sum_{j=1}^{m} S\left(\left(\pi_{j-1}, \pi_{j}\right]\right) \widetilde{\sigma}_{\pi_{j}}(T((r, s])) \in I_{\mathrm{h}} \otimes B\left(\mathcal{F}_{q+s)}\right) \otimes I_{[q+s},
\end{aligned}
$$

where $\pi=\left\{p=\pi_{0}<\cdots<\pi_{m}=q\right\}$ is a typical finite partition of the interval $[p, q]$.

Lemma 3.4 Suppose $S$ and T are finite quantum stop times, with $T$ discrete, so that there exists a finite set $\left\{t_{1}<\cdots<t_{m}\right\} \subseteq(0, \infty)$ such that $T\left(\left\{t_{1}, \ldots, t_{m}\right\}\right)=I$. Then

$$
(S \star T)(C)=\sum_{j=1}^{m} S\left(\left(C-t_{j}\right)_{+}\right) \sigma_{S}\left(T\left(\left\{t_{j}\right\}\right)\right) \quad \text { for all } C \in \mathcal{B}\left(\mathbb{R}_{+}\right),
$$

where $(C-t)_{+}:=\left\{s \in \mathbb{R}_{+}: s+t \in C\right\}$ for all $t \in \mathbb{R}_{+}$. 
Proof. Note first that, by Lemma 3.2,

$$
\sum_{j=1}^{m}(S \otimes T)\left(\mathbb{R}_{+} \times\left\{t_{j}\right\}\right)=\sum_{j=1}^{m} S\left(\mathbb{R}_{+}\right) \sigma_{S}\left(T\left(\left\{t_{j}\right\}\right)\right)=\sigma_{S}\left(T\left(\left\{t_{1}, \ldots, t_{m}\right\}\right)\right)=I,
$$

so

$$
\begin{aligned}
(S \star T)(C) & =(S \otimes T)\left(\left\{(x, y) \in \mathbb{R}_{+}^{2}: x+y \in C\right\}\right) \\
& =\sum_{j=1}^{m}(S \otimes T)\left(\left\{\left(x, t_{j}\right) \in \mathbb{R}_{+}^{2}: x+t_{j} \in C\right\}\right) \\
& =\sum_{j=1}^{m} S\left(\left(C-t_{j}\right)_{+}\right) \sigma_{S}\left(T\left(\left\{t_{j}\right\}\right)\right) .
\end{aligned}
$$

REMARK 3.5 If the discrete stopping time $T$ is supported at one point, so that $T(\{t\})=I$ for some $t \in(0, \infty)$, then $S \star T=S+t$, where $(S+t)(A):=S\left((A-t)_{+}\right)$for all $A \in \mathcal{B}[0, \infty]$.

Definition 3.6 Let $p \in B(\mathrm{k})$ be an orthogonal projection and, for all $t \in \mathbb{R}_{+}$, let $P_{[t} \in B\left(\mathcal{F}_{[t}\right)$ be the orthogonal projection such that $P_{[t} \varepsilon(f)=\varepsilon(p f)$ for all $f \in L^{2}([t, \infty)$; k), where $p$ acts pointwise.

A family of bounded operators $V=\left(V_{t}\right)_{t \in \mathbb{R}_{+}} \subseteq B(\mathrm{~h} \otimes \mathcal{F})$ is $p$-adapted if

$$
V_{t}=V_{t)} \otimes P_{[t} \quad \text { for every } t \in \mathbb{R}_{+},
$$

where $V_{t} \in B\left(\mathrm{~h} \otimes \mathcal{F}_{t}\right)$. If $p=0$ or $p=I_{\mathrm{k}}$ then $p$ adaptedness is known as vacuum adaptedness or identity adaptedness, respectively.

Given a $p$-adapted family of bounded operators $V$, the identity-adapted projection $\widehat{V}$ is the family of operators $\widehat{V}$, where $\widehat{V}_{t}:=V_{t} \otimes I_{[t}$ for all $t \in \mathbb{R}_{+}$.

A $p$-adapted family of bounded operators $V$ is an isometric cocycle if $\widehat{V}_{t}$ is an isometry for all $t \in \mathbb{R}_{+}$and

$$
V_{s+t}=\widehat{V}_{s} \widetilde{\sigma}_{s}\left(V_{t}\right) \quad \text { for all } s, t \in \mathbb{R}_{+}
$$

A $p$-adapted isometric cocycle $V$ is strongly continuous if $t \mapsto V_{t} z$ is continuous for all $z \in \mathrm{h} \otimes \mathcal{F}$.

Theorem 3.7 [2, Theorems 6.5 and 7.2, and Corollary 6.6] If $S$ is a finite quantum stop time, $V$ is a strongly continuous isometric p-adapted cocycle and

$$
V_{S, \pi}:=\sum_{k=1}^{n+1} V_{\pi_{k}} S\left(\left(\pi_{k-1}, \pi_{k}\right]\right)
$$

for any finite partition $\pi=\left\{0=\pi_{0}<\cdots<\pi_{n+1}=t\right\}$ of $[0, t]$, then $V_{S, \pi}$ is a contraction and there exists a contraction $V_{S, t} \in B(\mathrm{~h} \otimes \mathcal{F})$ such that $V_{S, \pi} \rightarrow V_{S, t}$ in the strong operator topology 
as $\pi$ is refined, for all $t \in(0, \infty)$. Furthermore, there exists a contraction $V_{S} \in B(\mathrm{~h} \otimes \mathcal{F})$ such that $V_{S, t} \rightarrow V_{S}$ in the strong operator topology as $t \rightarrow \infty$, and

$$
V_{S+t}=\widehat{V}_{S} \widetilde{\sigma}_{S}\left(V_{t}\right) \quad \text { for all } t \in \mathbb{R}_{+}
$$

Proof. The only thing which not immediate is the assertion, at end of [2, Proof of Theorem 7.2], that

$$
\widehat{V}_{S, \pi \cap[0, t]} \widetilde{\sigma}_{S, \pi}(Z) \rightarrow \widehat{V}_{S, t} \widetilde{\sigma}_{S}(Z)
$$

as the partition $\pi$ is refined, for all $Z \in B(\mathrm{~h} \otimes \mathcal{F})$ and $t \geq 0$. (In fact, a very slightly weaker claim is made.) It follows from [2, Theorem 6.5] that

$$
\widehat{V}_{S, \pi \cap[0, t]} \rightarrow \widehat{V}_{S, t}
$$

in the strong operator topology, and $\left\|\widehat{V}_{S, \pi \cap[0, t]}\right\| \leq 1$ for all $\pi$, by [2, Lemma 6.4], so the claim holds as long as $\widetilde{\sigma}_{S, \pi}(Z) \rightarrow \widetilde{\sigma}_{S}(Z)$ in the strong operator topology. However, this is part of Theorem 2.9.

LEMMA 3.8 If $S$ and $T$ are finite quantum stop times, with $T$ discrete, then

$$
V_{S \star T}=\widehat{V}_{S} \widetilde{\sigma}_{S}\left(V_{T}\right)
$$

for any strongly continuous isometric p-adapted cocycle $V$.

Proof. If $T$ is as in the statement of Lemma 3.4 and $t>t_{m}$, then

$$
\begin{aligned}
V_{S \star T, t} & =\underset{\pi}{\operatorname{st} . \lim _{\pi}} \sum_{k=1}^{n+1} V_{\pi_{k}}(S \star T)\left(\left(\pi_{k-1}, \pi_{k}\right]\right) \\
& =\underset{\pi}{\operatorname{st} . \lim } \sum_{j=1}^{m} \sum_{k=1}^{n+1} V_{\pi_{k}} S\left(\left(\pi_{k-1}-t_{j}, \pi_{k}-t_{j}\right]_{+}\right) \widetilde{\sigma}_{S}\left(T\left(\left\{t_{j}\right\}\right)\right),
\end{aligned}
$$

where $\pi=\left\{0=\pi_{0}<\cdots<\pi_{n+1}=t\right\}$ and $(x, y]_{+}=\left\{s \in \mathbb{R}_{+}: x<s \leq y\right\}$. For $j=1, \ldots, m$ and $k=0, \ldots, n+1$, let

$$
\pi_{k}^{j}= \begin{cases}\pi_{k}-t_{j} & \text { if } \pi_{k} \geq t_{j} \\ 0 & \text { otherwise }\end{cases}
$$

so that $\pi^{j}$ is a partition of $\left[0, t-t_{j}\right]$. Then 


$$
\begin{aligned}
\sum_{j=1}^{m} & \sum_{k=1}^{n+1} V_{\pi_{k}} S\left(\left(\pi_{k-1}-t_{j}, \pi_{k}-t_{j}\right]_{+}\right) \widetilde{\sigma}_{S}\left(T\left(\left\{t_{j}\right\}\right)\right) \\
= & \sum_{j=1}^{m} \sum_{k=1}^{n+1} V_{\pi_{k}^{j}+t_{j}} S\left(\left(\pi_{k-1}^{j}, \pi_{k}^{j}\right]\right) \widetilde{\sigma}_{S}\left(T\left(\left\{t_{j}\right\}\right)\right) \\
= & \sum_{j=1}^{m} \sum_{k=1}^{n+1} \widehat{V}_{\pi_{k}^{j}} S\left(\left(\pi_{k-1}^{j}, \pi_{k}^{j}\right]\right) \sum_{l=1}^{n+1} S\left(\left(\pi_{l-1}^{j}, \pi_{l}^{j}\right]\right) \widetilde{\sigma}_{\pi_{l}^{j}}\left(V_{t_{j}}\right) \widetilde{\sigma}_{S}\left(T\left(\left\{t_{j}\right\}\right)\right) \\
& \rightarrow \sum_{j=1}^{m} \widehat{V}_{S, t-t_{j}} \widetilde{\sigma}_{S}\left(V_{t_{j}}\right) \widetilde{\sigma}_{S}\left(T\left(\left\{t_{j}\right\}\right)\right)
\end{aligned}
$$

in the strong operator topology as $\pi$ is refined; for the final identity, note that

$$
V_{s+t} S((r, s])=\widehat{V_{s}} \widetilde{\sigma}_{s}\left(V_{t}\right) S((r, s])=\widehat{V}_{s} S((r, s]) \widetilde{\sigma}_{s}\left(V_{t}\right) \quad \text { whenever } \quad 0 \leq r<s<t<\infty
$$

Hence

$$
V_{S \star T}=\operatorname{st}_{t \rightarrow \infty} V_{S \star T, t}=\operatorname{st}_{t \rightarrow \infty} \sum_{j=1}^{m} \widehat{V}_{S, t-t_{j}} \widetilde{\sigma}_{S}\left(V_{t_{j}} T\left(\left\{t_{j}\right\}\right)\right)=\widehat{V}_{S} \widetilde{\sigma}_{S}\left(V_{T}\right) .
$$

Definition 3.9 (Cf. [7, p. 322]) A sequence of finite quantum stop times $\left(S_{n}\right)_{n \geq 1}$ is said to converge to a quantum stop time $S$, written $S_{n} \Rightarrow S$, if $S_{n}([0, t]) \rightarrow S([0, t])$ in the strong operator topology for all but a countable set of points $t \in \mathbb{R}_{+}$.

LEMMA 3.10 Let $V$ be a strongly continuous isometric p-adapted cocycle. If $\left(S_{n}\right)_{n \geq 1}$ is a sequence of finite quantum stop times such that $S_{n} \Rightarrow S$ for some finite quantum stop time $S$, then $V_{S_{n}} \rightarrow V_{S}$ in the strong operator topology.

Proof. The usual approximation argument shows it suffices to prove that $\left\|\left(V_{S_{n}}-V_{S}\right) u \varepsilon(f)\right\| \rightarrow 0$ as $n \rightarrow \infty$, where $u \in \mathrm{h}$ and $f \in L^{2}\left(\mathbb{R}_{+} ; \mathrm{k}\right)$ are arbitrary.

From the proof of [2, Corollary 6.6], if $S$ is any finite quantum stop time and $s, t \in \mathbb{R}_{+}$are such that $s \leq t$, then

$$
\left\|\left(V_{S, t}-V_{S, s}\right) u \varepsilon(f)\right\| \leq\|S((s, t]) u \varepsilon(f)\| .
$$

Letting $t \rightarrow \infty$ and recalling that $S(\{\infty\})=0$, it follows that

$$
\left\|\left(V_{S}-V_{S, s}\right) u \varepsilon(f)\right\| \leq\|S((s, \infty)) u \varepsilon(f)\| .
$$

Furthermore, from the proof of [2, Theorem 6.5],

$$
\left\|\left(V_{S, \pi^{\prime}}-V_{S, \pi}\right) u \varepsilon(f)\right\| \leq \sup \left\{\left\|\left(V_{r}-V_{\pi_{j}}\right) u \varepsilon(f)\right\|: r \in\left[\pi_{j}, \pi_{j+1}\right], j=0, \ldots, m\right\}\|S([0, s]) \varepsilon(f)\|,
$$

where $\pi^{\prime}$ is any refinement of the partition $\pi=\left\{0=\pi_{0}<\cdots<\pi_{m+1}=s\right\}$; refining $\pi^{\prime}$ shows that the same inequality holds with $V_{S, \pi^{\prime}}$ replaced by $V_{S, s}$. Hence 


$$
\begin{aligned}
& \left\|\left(V_{S}-V_{S, \pi}\right) u \varepsilon(f)\right\| \\
& \quad \leq \sup \left\{\left\|\left(V_{r}-V_{\pi_{j}}\right) u \varepsilon(f)\right\|: r \in\left[\pi_{j}, \pi_{j+1}\right], j=0, \ldots, m\right\}\|\varepsilon(f)\|+\|S((s, \infty)) u \varepsilon(f)\| .
\end{aligned}
$$

Now fix $\varepsilon>0$, choose $s \in \mathbb{R}_{+}$such that $S_{n}([0, s]) \rightarrow S([0, s])$ in the strong operator topology and $\|S((s, \infty)) u \varepsilon(f)\|<\varepsilon$, and note that $\left\|S_{n}((s, \infty)) u \varepsilon(f)\right\|<\varepsilon$ for all sufficiently large $n$. Therefore,

$$
\begin{aligned}
\left\|\left(V_{S}-V_{S_{n}}\right) u \varepsilon(f)\right\| \leq & \left\|\left(V_{S}-V_{S, \pi}\right) u \varepsilon(f)\right\|+\left\|\left(V_{S_{n}}-V_{S_{n}, \pi}\right) u \varepsilon(f)\right\|+\left\|\left(V_{S, \pi}-V_{S_{n}, \pi}\right) u \varepsilon(f)\right\| \\
& <2 \sup \left\{\left\|\left(V_{r}-V_{\pi_{j}}\right) u \varepsilon(f)\right\|: r \in\left[\pi_{j}, \pi_{j+1}\right], j=0, \ldots, m\right\}\|\varepsilon(f)\| \\
& \quad+2 \varepsilon+\left\|\left(V_{S, \pi}-V_{S_{n}, \pi}\right) u \varepsilon(f)\right\| \\
& <4 \varepsilon+\left\|\left(V_{S, \pi}-V_{S_{n}, \pi}\right) u \varepsilon(f)\right\|
\end{aligned}
$$

as long as $\pi$ is chosen to be sufficiently fine, so that

$$
\sup \left\{\left\|\left(V_{r}-V_{\pi_{j}}\right) u \varepsilon(f)\right\|: r \in\left[\pi_{j}, \pi_{j+1}\right], j=0, \ldots, m\right\}\|\varepsilon(f)\|<\varepsilon .
$$

Finally, if $\pi$ is chosen so $S_{n}\left(\left[0, \pi_{j}\right]\right) \rightarrow S\left(\left[0, \pi_{j}\right]\right)$ in the strong operator topology as $n \rightarrow \infty$, for $j=0, \ldots, m+1$, then, since

$$
\left\|\left(V_{S, \pi}-V_{S_{n}, \pi}\right) u \varepsilon(f)\right\| \leq \sum_{j=1}^{m+1}\left\|V_{\pi_{j}}\left(S\left(\pi_{j-1}, \pi_{j}\right]\right)-\left(S_{n}\left(\pi_{j-1}, \pi_{j}\right]\right) u \varepsilon(f)\right\| \rightarrow 0
$$

as $n \rightarrow \infty$, and $\varepsilon$ is arbitrary, the result follows.

LEMMA 3.11 Let $T$ be a finite quantum stop time, and suppose that the multiplicity space $\mathrm{k}$ is separable. There exists a sequence of discrete quantum stop times $\left(T_{n}\right)_{n \geq 1}$ such that $T_{n} \Rightarrow T$. Furthermore, $S \star T_{n} \Rightarrow S \star T$ for any finite quantum stop time $S$.

Proof. As is well known, a spectral measure is strongly right continuous with left limits: if $x \in \mathcal{F}$, then

$$
\lim _{s \rightarrow t+} S([0, t]) x-S([0, s]) x=-\lim _{s \rightarrow t+} S((t, s]) x=0,
$$

whereas

$$
\lim _{s \rightarrow t-} S([0, t]) x-S([0, s]) x=\lim _{s \rightarrow t-} S((s, t]) x=S(\{t\}) x
$$

In particular, the set of discontinuities $\mathcal{D}_{S}(x):=\left\{t \in \mathbb{R}_{+}: S(\{t\}) x \neq 0\right\}$ is countable.

Now suppose $\left\{x_{n}: n \geq 1\right\}$ is dense in $\mathcal{F}$ and let $\mathcal{D}_{S}:=\cup_{n \geq 1} \mathcal{D}_{S}\left(x_{n}\right)$. An $\varepsilon / 3$ argument shows that $t \mapsto S([0, t]) x$ is continuous on $\mathbb{R}_{+} \backslash \mathcal{D}_{S}$ for all $x \in \mathcal{F}$, so $\mathcal{D}_{S}$ is the set of discontinuities of $S$ on $\mathbb{R}_{+}$.

For all $n \geq 1$, let the finite partition $\pi^{n}=\left\{0=\pi_{0}^{n}<\pi_{1}^{n}<\cdots<\pi_{n}^{n}<\infty\right\}$ be such that $\pi_{n}^{n} \rightarrow \infty$ and $\max \left\{\pi_{k}^{n}-\pi_{k-1}^{n}: k=1, \ldots, n\right\} \rightarrow 0$ as $n \rightarrow \infty$. Define a discrete quantum stop time 


$$
T_{n}: \mathcal{B}[0, \infty] \rightarrow B(\mathcal{F}) ; A \mapsto \sum_{k=1}^{n-1} 1_{A}\left(\pi_{k}^{n}\right) T\left(\left(\pi_{k-1}^{n}, \pi_{k}^{n}\right]\right)+1_{A}\left(\pi_{n}^{n}\right) T\left(\left(\pi_{n}^{n}, \infty\right]\right),
$$

and note that

$$
T_{n}([0, t])= \begin{cases}0 & \text { if } 0 \leq t<\pi_{1}^{n}, \\ T\left(\left[0, \pi_{1}^{n}\right]\right) & \text { if } \pi_{1}^{n} \leq t<\pi_{2}^{n}, \\ \vdots & \vdots \\ T\left(\left[0, \pi_{n-1}^{n}\right]\right) & \text { if } \pi_{n-1}^{n} \leq t<\pi_{n}^{n}, \\ I & \text { if } \pi_{n}^{n} \leq t \leq \infty .\end{cases}
$$

Thus, if $x \in \mathcal{F}$ and $t \in \mathbb{R}_{+}$then $\pi_{k}^{n}>t \geq \pi_{k-1}^{n}$ for some $k \in\{1, \ldots, n\}$ once $n$ is sufficiently large, so

$$
T([0, t]) x-T_{n}([0, t]) x=T\left(\left(\pi_{k-1}^{n}, t\right]\right) x \rightarrow T(\{t\}) x
$$

as $n \rightarrow \infty$. This last term equals 0 if $t \in \mathbb{R}_{+} \backslash \mathcal{D}_{T}$, and thus $T_{n} \Rightarrow T$.

For the final claim, let $S$ be a finite quantum stop time. If $t \in \mathbb{R}_{+}$and $f \in L^{2}\left(\mathbb{R}_{+}\right.$; k) then, by [2, Corollary 3.5],

$$
\begin{aligned}
I_{n}(t):= & \left\|\left(\left(S \star T_{n}\right)([0, t])-(S \star T)([0, t])\right) \varepsilon(f)\right\|^{2} \\
& =\int_{[0, t]}\left\|\left(T-T_{n}\right)([0, t-s]) \Gamma_{s}^{*} \varepsilon(f)\right\|^{2} \exp \left(-\int_{s}^{\infty}\|f(u)\|^{2} \mathrm{~d} u\right)\|S(\mathrm{~d} s) \varepsilon(f)\|^{2} .
\end{aligned}
$$

To prove that $S \star T_{n} \Rightarrow S \star T$, it suffices to show that $I_{n}(t) \rightarrow 0$ as $n \rightarrow \infty$ for all but countably many $t \in \mathbb{R}_{+}$, by the usual approximation argument.

Now, as $n \rightarrow \infty$, so $\left(T-T_{n}\right)([0, t-s]) \rightarrow T(\{t-s\})$, by the previous working. Thus, the dominated convergence theorem gives that

$$
I_{n}(t) \rightarrow I(t):=\sum_{r \in \mathcal{D} \cap[0, t]}\left\|T(\{r\}) \Gamma_{t-r}^{*} \varepsilon(f)\right\|^{2} \exp \left(-\int_{t-r}^{\infty}\|f(u)\|^{2} \mathrm{~d} u\right)\|S(\{t-r\}) \varepsilon(f)\|^{2} .
$$

Thus, $I(t)=0$ whenever $t \notin \mathcal{D}_{S}+\mathcal{D}_{T}:=\left\{s+r: s \in \mathcal{D}_{S}, r \in \mathcal{D}_{T}\right\}$ and the result follows.

REMARK 3.12 If the multiplicity space $\mathrm{k}$ is not separable, the statement of [2, Corollary 3.5] requires strong measurability, not just Borel measurability, of $F$ and $G$. As $t \mapsto \Gamma_{t}$ and $t \mapsto \Gamma_{t}^{*}$ are strongly continuous and $t \mapsto S([0, t])$ is strongly right continuous on $\mathbb{R}_{+}$, all the subsequent proofs in [2] remain valid.

REMARK 3.13 It is straightforward to construct on a non-separable Hilbert space a spectral measure which has an uncountable set of discontinuities. Thus, the separability hypothesis in Lemma 3.11 may not be dropped.

THEOREM 3.14 Let $V$ be a strongly continuous isometric p-adapted cocycle, and suppose that the multiplicity space $\mathrm{k}$ is separable. If $S$ and $T$ are finite quantum stop times, then 


$$
V_{S \star T}=\widehat{V}_{S} \widetilde{\sigma}_{S}\left(V_{T}\right) .
$$

Proof. By Lemma 3.11, there exists a sequence of discrete quantum stop times $\left(T_{n}\right)_{n \geq 1}$ such that $T_{n} \Rightarrow T$ and $S \star T_{n} \Rightarrow S \star T$. Hence $V_{S \star T_{n}} \rightarrow V_{S \star T}$ in the strong operator topology, by Lemma 3.10. Furthermore, $V_{S \star T_{n}}=V_{S} \widetilde{\sigma}_{S}\left(V_{T_{n}}\right)$ for all $n \geq 1$, by Lemma 3.8, so the result follows from another application of Lemma 3.10 together with Remark 2.10, that $\sigma_{S}$ is strong operator continuous on bounded sets.

The next two theorems show that stopping an isometric cocycle can be used to produce a form of inner non-unital Evans-Hudson flow.

THEOREM 3.15 Let V be a strongly continuous isometric identity-adapted cocycle. The map

$$
\alpha_{S}: B(\mathrm{~h}) \rightarrow B(\mathrm{~h} \otimes \mathcal{F}) ; a \mapsto V_{S}(a \otimes I) V_{S}^{*}
$$

is a *-homomorphism for any finite quantum stop time S. Furthermore, if the multiplicity space $\mathrm{k}$ is separable, the identity

$$
\alpha_{S \star T}=\widehat{\alpha}_{S} \circ \widetilde{\sigma}_{S} \circ \alpha_{T}
$$

holds for any finite quantum stop times $S$ and $T$, where

$$
\widehat{\alpha}_{S}: B(\mathrm{~h} \otimes \mathcal{F}) \rightarrow B(\mathrm{~h} \otimes \mathcal{F}) ; X \mapsto V_{S} X V_{S}^{*}
$$

Proof. Note that $V_{S}^{*} V_{S}=I_{\mathrm{h}} \otimes I$, by [2, Proposition 6.8]. Thus if $a, b \in B(\mathrm{~h})$, then

$$
\alpha_{S}(a) \alpha_{S}(b)=V_{S}(a \otimes I) V_{S}^{*} V_{S}(b \otimes I) V_{S}^{*}=V_{S}(a b \otimes I) V_{S}=\alpha_{S}(a b)
$$

so $\alpha_{S}$ is multiplicative. Linearity and $*$-preservation are immediate.

For the second claim, note that $\widehat{V}=V$. Hence, by Theorem 3.14, if $a \in B(\mathrm{~h})$, then

$$
\begin{aligned}
\alpha_{S \star T}(a)=V_{S \star T}(a \otimes I) V_{S \star T}^{*} & =V_{S} \widetilde{\sigma}_{S}\left(V_{T}\right)(a \otimes I) \widetilde{\sigma}_{S}\left(V_{T}^{*}\right) V_{S}^{*} \\
& =V_{S} \widetilde{\sigma}_{S}\left(V_{T}(a \otimes I) V_{T}^{*}\right) V_{S}^{*} \\
& =\left(\widehat{\alpha}_{S} \circ \widetilde{\sigma}_{S} \circ \alpha_{T}\right)(a) ;
\end{aligned}
$$

the penultimate equality holds because $\sigma_{S}$ is unital, so $\widetilde{\sigma}_{S}(a \otimes I)=a \otimes I$.

THEOREM 3.16 Let $V$ be a strongly continuous isometric vacuum-adapted cocycle. The map

$$
\beta_{S}: B(\mathrm{~h}) \rightarrow B(\mathrm{~h} \otimes \mathcal{F}) ; a \mapsto V_{S}\left(a \otimes E_{S}\right) V_{S}^{*}
$$

is a *-homomorphism for any finite quantum stop time $S$. Furthermore, if the multiplicity space $\mathrm{k}$ is separable, the identity 


$$
\beta_{S \star T}=\widehat{\beta_{S}} \circ \widetilde{\sigma}_{S} \circ \beta_{T}
$$

holds for any finite quantum stop times $S$ and $T$, where

$$
\widehat{\beta_{S}}: B(\mathrm{~h} \otimes \mathcal{F}) \rightarrow B(\mathrm{~h} \otimes \mathcal{F}) ; X \mapsto \widehat{V_{S}} X \widehat{V}_{S}^{*}
$$

Proof. Note that $V_{S}^{*} V_{S}=\widetilde{E}_{S}$, by [2, Proposition 6.7]. Thus if $a, b \in B(\mathrm{~h})$, then

$$
\beta_{S}(a) \beta_{S}(b)=V_{S}\left(a \otimes E_{S}\right) V_{S}^{*} V_{S}\left(b \otimes E_{S}\right) V_{S}^{*}=V_{S}\left(a b \otimes E_{S}\right) V_{S}=\beta_{S}(a b),
$$

so $\beta_{S}$ is multiplicative. As above, linearity and $*$-preservation are immediate.

For the second claim, let $a \in B(\mathrm{~h})$ and note that, by Theorem 3.14,

$$
\begin{aligned}
\beta_{S \star T}(a)=V_{S \star T}\left(a \otimes E_{S \star T}\right) V_{S \star T}^{*} & =\widehat{V}_{S} \widetilde{\sigma}_{S}\left(V_{T}\right)\left(a \otimes E_{S \star T}\right) \widetilde{\sigma}_{S}\left(V_{T}^{*}\right) \widehat{V}_{S}^{*} \\
& =\widehat{V}_{S} \widetilde{\sigma}_{S}\left(V_{T}\left(a \otimes E_{T}\right) V_{T}^{*}\right) \widehat{V}_{S}^{*} \\
& =\left(\widehat{\beta}_{S} \circ \widetilde{\sigma}_{S} \circ \beta_{T}\right)(a) ;
\end{aligned}
$$

for the penultimate equality, note that $E_{S \star T}=\sigma_{S}\left(E_{T}\right)$, by [2, Theorem 5.4], which implies immediately that $\widetilde{\sigma}_{S}\left(a \otimes E_{T}\right)=a \otimes E_{S \star T}$.

REMARK 3.17 In the context of Theorems 3.15 and 3.16, note that $\alpha_{S}\left(I_{h}\right)=V_{S} V_{S}^{*}=\beta_{S}\left(I_{h}\right)$. The former identity is immediate, and the latter holds because $V_{S, \pi} \widetilde{E}_{S, \pi^{\prime}} V_{S, \pi}=V_{S, \pi} V_{S, \pi}^{*}$ if $V$ is vacuum adapted, where $\pi$ is any finite partition of $[0, t]$ and $\pi^{\prime}$ is its one-point extension to a partition of $[0, \infty]$.

REMARK 3.18 If the finite quantum stop time $S$ is deterministic, so that $S(\{s\})=I$ for some $s \in(0, \infty)$, then $V_{S}=V_{s}$ and $\widetilde{\sigma}_{S}=\widetilde{\sigma}_{s}$. It follows that (5) and (6) are the stop-time generalization of the deterministic mapping-cocycle relation [3]

$$
\alpha_{s+t}=\widehat{\alpha}_{s} \circ \widetilde{\sigma}_{s} \circ \alpha_{t} \quad \text { for all } s, t \geq 0 .
$$

\section{Acknowledgements}

This work was begun during a conference held at the Kerala School of Mathematics, Kozhikode, India; the warm hospitality and stimulating atmosphere provided by the organizers is gratefully acknowledged. It continued during visits of the first author to the Indian Statistical Institute, Kolkata, and the Jawaharlal Nehru Centre for Advanced Scientific Research, Bangalore, with travel supported by the ANCM project of the Indian Statistical Institute. The second author gratefully acknowledges support by a grant from the SERB-Distinguished Fellowship of the Department of Science and Technology, Government of India. 


\section{References}

1. D. Applebaum, Stopping unitary processes in Fock space, Publ. RIMS Kyoto Univ. 24 (1988), 697-705.

2. A. C. R. Belton and K. B. Sinha, Stopping the CCR flow and its isometric cocycles, Q. J. Math. 65 (2014), 1145-1164

3. W. S. Bradshaw, Stochastic cocycles as a characterisation of quantum flows, Bull. Sci. Math. 116 (1992), 1-34.

4. R. L. Hudson, The strong Markov property for canonical Wiener processes, J. Funct. Anal. 34 (1979), 266-281.

5. R. L. Hudson, Stop times in Fock space quantum probability, Stochastics 79 (2007), 383-391.

6. R. L. Hudson, Stopping Weyl Processes, Recent Development in Stochastic Dynamics and Stochastic Analysis, J. Duan, S. Luo and C. Wang Interdisciplinary Mathematical Sciences 8. World Scientific, Singapore, 2010, 185-193.

7. K. R. Parthasarathy and K. B. Sinha, Stop times in Fock space stochastic calculus, Probab. Theory Relat. Fields 75 (1987), 317-349.

8. M. A. Pinsky, Stochastic integral representation of multiplicative operator functionals of a Wiener process, Trans. Amer. Math. Soc. 167 (1972), 89-104. 